\title{
The Treatment of Hypersalivation in Rett Syndrome with Botulinum Toxin: Efficacy and Clinical Implications
}

\author{
Pia Bernardo (D) - Enza Raiano - Gerarda Cappuccio • Raffaele Dubbioso • \\ Carmela Bravaccio · Emilia Vergara - Silvio Peluso · Fiore Manganelli • \\ Marcello Esposito
}

Received: September 28, 2018 / Published online: January 8, 2019

(C) The Author(s) 2019

\section{ABSTRACT}

Introduction: Subjects with Rett syndrome (RS) develop invariably severe motor deterioration resulting in swallowing difficulties that may produce excessive drooling. Hypersalivation can cause discomfort due to hygienic problems

Pia Bernardo and Enza Raiano contributed equally to this work.

Enhanced digital features To view enhanced digital features for this article, go to https://doi.org/10.6084/ m9.figshare.7498991.

Electronic supplementary material The online version of this article (https://doi.org/10.1007/s40120018-0125-9) contains supplementary material, which is available to authorized users.

P. Bernardo $(\varangle) \cdot$ G. Cappuccio $~ C$. Bravaccio Department of Translational Medical Sciences, Federico II University, Naples, Italy

e-mail: pia.bernardo84@gmail.com

P. Bernardo

Department of Pediatric Neurosciences,

Neuropsychiatry Unit, Santobono-Pausilipon

Children's Hospital, Naples, Italy

E. Raiano - R. Dubbioso - S. Peluso - F. Manganelli ·

M. Esposito

Department of Neurosciences, Reproductive

Sciences and Odontostomatology, University

Federico II of Naples, Naples, Italy

E. Vergara

Department of Advanced Biomedical Sciences,

University of Naples Federico II, Naples, Italy and may complicate with oral and respiratory dysfunctions. The aim of this study is to evaluate the response to treatment with botulinum toxin (BTX) for hypersalivation and to identify possible benefits of saliva reduction on oral motor and respiratory disorders of patients with RS.

Methods: Five consecutive patients with RS and hypersalivation were treated with incobotulinumtoxin A injected in salivary glands with ultrasound guidance. Severity of excessive drooling was assessed with the Thomas-Stonell and Greenberg Scale (TGSC) and the clinical impact of the treatment was evaluated using three selected items of RS Assessment Rating Scale (R.A.R.S.): eating habits, dyspnoea and bruxism. Scale rating was performed before BTX injection (T0), 4 (T1) and 12 (T2) weeks after.

Results: Scores of TGSC and R.A.R.S. (for eating and bruxism) were reduced significantly after therapy at $\mathrm{T} 1$.

Conclusions: BTX treatment for sialorrhea in $\mathrm{RS}$ is effective in reducing saliva production and may also improve oral motor functions.

Keywords: Botulinum toxin; Bruxism; Drooling; Rett syndrome

\section{INTRODUCTION}

Rett syndrome (RS) is a severe rare neurodevelopment disorder, affecting almost exclusively 
females and presenting with psychomotor regression and autistic features [1]. RS diagnosis is based on clinical criteria and is supported by genetic testing. Loss-of-function mutations of the gene-encoding methyl-CpG-binding protein 2 (MECP2) are usually associated with RS [2].

Excessive drooling frequently impairs quality of life in children with RS. Hypersalivation is probably secondary to swallowing difficulties and it can be worsened by other factors, like antiepileptic drug (AED) intake and respiratory problems [3]. Drooling is also reported in several autistic syndromes associated with neurologic and metabolic disease, like mitochondrial dysfunctions or in Niemann-Pick type C [4]. The occurrence of hypersalivation in RS was reported only in a few and dated studies. A survey of RS in North America conducted by Coleman and colleagues 30 years ago found that drooling was present in $84 \%$ of patients with RS [5].

Botulinum toxin (BTX) injection in salivary glands is reported to be effective to reduce saliva production both in adults with neurodegenerative disorders and in children with cerebral palsy (CP). RS patients share several clinical features with children affected by CP; therefore, it is reasonable that BTX treatment of sialorrhea could be effective also for them [6].

The aim of this study is to assess the efficacy and safety of BTX injections for excessive drooling in a group of patients with RS and to identify possible further benefits from the treatment.

\section{METHODS}

Five consecutive female patients affected by RS with excessive drooling and never treated before with BTX were recruited from January to December 2017 at the Child Neuropsychiatric Department of "Federico II" University of Naples, Italy. Diagnosis of RS in each patient was based on clinical criteria and confirmed by the evidence of MECP2 gene deletions.

Patients were assessed for hypersalivation with the Thomas-Stonell and Greenberg Scale (TSGS) [7]. The TSGS is a semiquantitative assessment of drooling severity (five-point scale, from $0=$ no drooling to $5=$ profuse drooling) and frequency (four-point scale, $0=$ no drooling to $5=$ constant drooling).

The impact of excessive drooling on oral motor and respiratory features of the disease were evaluated with the Rett Assessment Rating Scale (RARS) [8] for the following three items: eating habits, dyspnoea and bruxism. The RARS provides extensive information about global clinical conditions of patients with RS. It is divided into seven domains: cognitive, sensorial, motor, emotional, autonomy, typical characteristics of the disease and behavior (fourpoint scale, $1=$ no symptoms to $4=$ multiple symptoms) [9].

BTX type A was injected in submandibular and parotid glands bilaterally with the aid of ultrasound guidance using a $7-12-\mathrm{MHz}$ linear probe. All treatments were performed using a fix total dose of one BTX brand in order to have a more reliable comparison on drug efficacy among patients: $150 \mathrm{U}$ of incobotulinumtoxin A $\left(\right.$ Xeomin $\left.{ }^{\mathrm{TM}}\right)$. The score of TSGS and RARS on the selected items was detected before BTX treatment (T0), 4 weeks (T1) and 12 weeks (T2) after the injections. A consent form was signed by one parent of each girl participating in the study, in accordance with the Declaration of Helsinki.

The occurrence of adverse events was assessed by a medical evaluation at T1 and T2 and interviewing parents by telephone 2 weeks after the injections.

\section{Statistical Analysis}

Given the small number of subjects and because the distribution of variables could not be assumed to be approximately normal, all statistical analyses applied were nonparametric tests. The effects of BTX on the outcome measures (TSGS and RARS on the selected items) over time were evaluated by means of Friedman tests. As post hoc analysis, we used the Wilcoxon signed rank test to evaluate the benefit from BTX injections after each injection. Data were analyzed using software (SPSS v. 22.0 for Windows; SPSS Inc.). The significance level was 
set as $p=0.05$. Alpha inflation due to multiple comparisons was controlled according to Bonferroni's approach when appropriate. Data are presented as median (interquartile range, IQR).

\section{RESULTS}

Patients included in the study were 5 females with age ranging from 13 to 23 years (median: $19 \pm 5.75)$. All patients utilized a wheelchair and were not able to speak. Four patients were receiving enteral nutrition by percutaneous gastrostomy with integration of oral nutritional supplements. Three patients were under AEDs. Clinical details are summarized in Table 1.

The TSGS score was reduced significantly after treatment $\left[\chi^{2}(2)=9.294, p=0.01\right.$, Friedman test], and these results were confirmed by post hoc analysis with Wilcoxon signed rank tests, putting forward a significant improvement of TSGS score after 4 weeks [T0: 4 (1) vs. T1: $2(0.25) ; p=0.016]$, with no significant effect after 12 weeks [T0: 4 (1) vs T2: 4 (0.25); $p=0.157]$. A clinical improvement after treatment was also observed for the RARS bruxism score $\left[\chi^{2}(2)=7.429, p=0.024\right.$, Friedman test $]$ and RARS eating score $\left[\chi^{2}(2)=6.000, p=0.049\right.$, Friedman test], but, unfortunately, such statistical significance did not survive after post hoc analysis ( $p>0.059$; Wilcoxon signed rank test). Lastly, a trend towards statistical significance was observed for the RARS dyspnea score after BTX injections $\left[\chi^{2}(2)=5.600, p=0.061\right.$, Friedman test; Fig. 1]. Scores of TSGS and RARS items are presented in supplemental materials (Table S1).

In one patient, swallowing difficulties worsened 2 weeks after BTX injections and recovered 4 weeks later, with no major complications resulted from dysphagia. No side effects or adverse events related to BTX treatment were noticed in the remaining patients.

\section{DISCUSSION}

This study shows that BTX injection into salivary glands can decrease saliva production, reducing excessive drooling in a small sample of

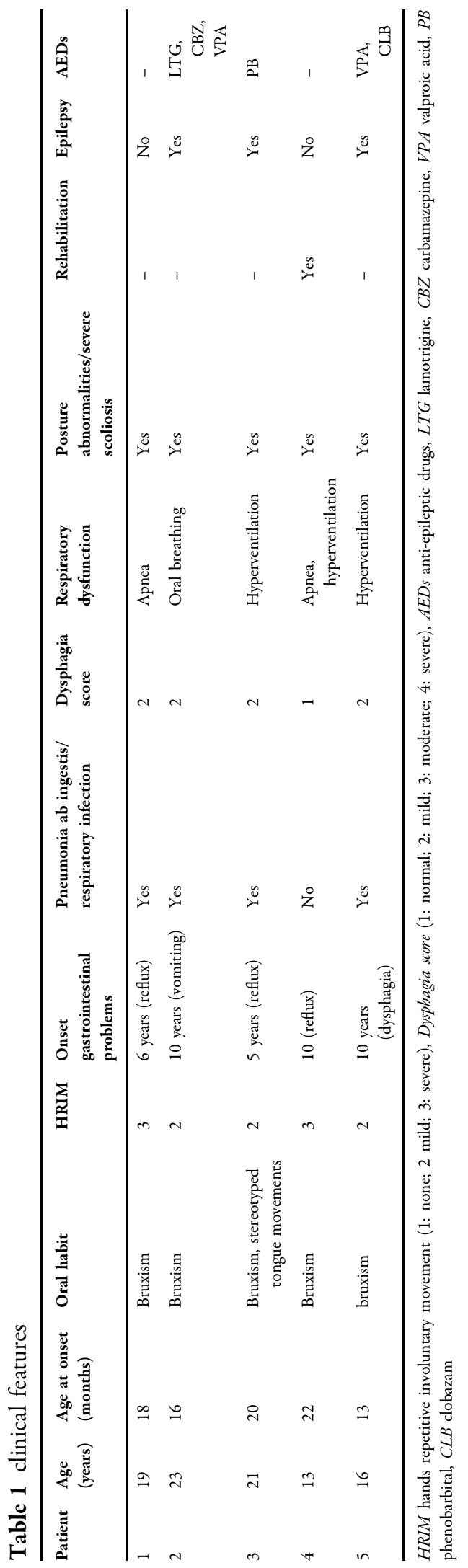




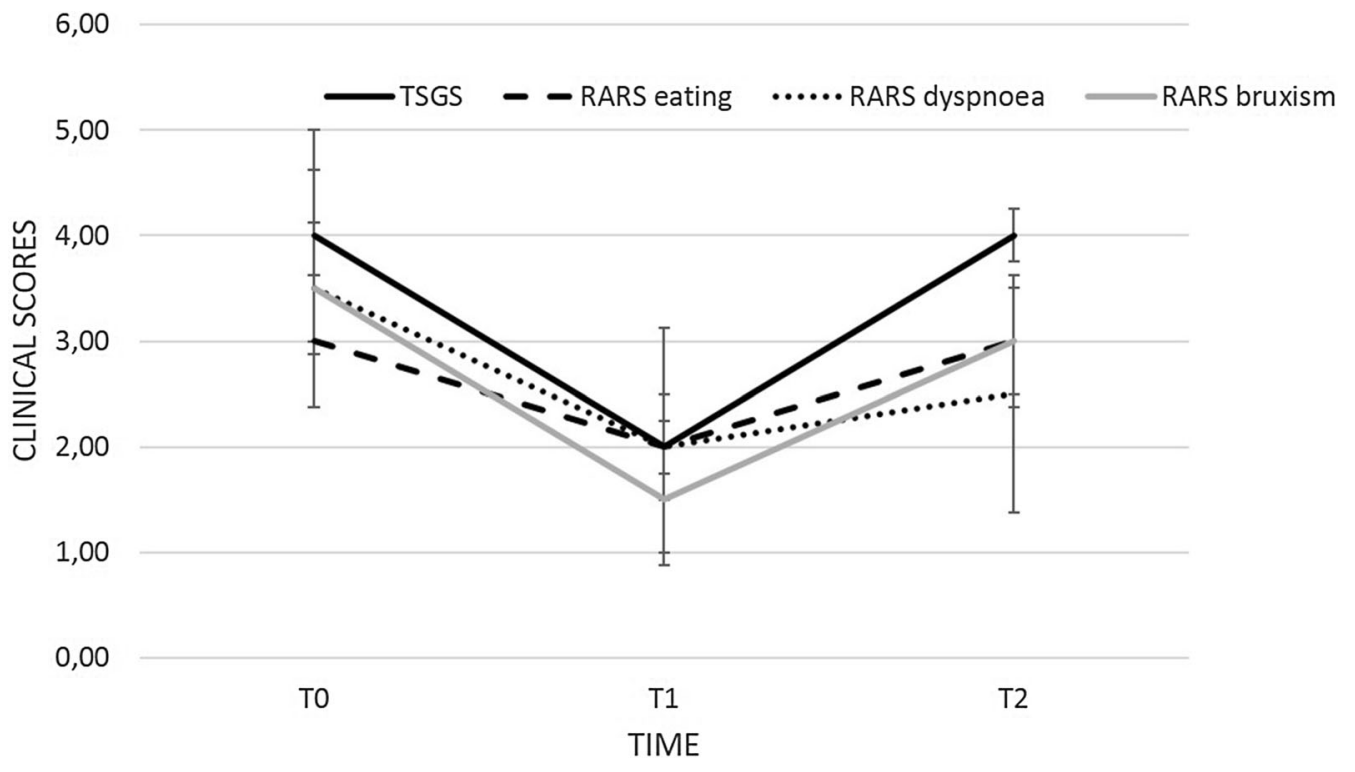

Fig. 1 Effect of BTX injections on clinical scores (TSGS, RARS eating, RARS dyspnoea, RARS bruxism) after 4 (T1) and 12 weeks (T2)

patients affected by RS. Improvement in hypersalivation seems also to affect positively a few RS clinical features related to oral motor dysfunctions.

Previous studies showed that BTX-A is effective in reducing drooling both in adults with neurodegenerative disorders and in children with CP [10]. This is the first report of BTX treatment for sialorrhea in children and young adults affected by a genetic disorder like RS. All patients were injected with the same brand of BTX, incobotulinumtoxin A, in order to have more reliable results for comparisons among patients. Patients received the dose of $150 \mathrm{U}$ that is higher in comparison to the amount reported in previous studies on the treatment of sialorrhea. When our study started, there was no official indication of BTX for excessive salivation and we chose to give that dose because, in our experience, it was effective and safe if injections were performed under ultrasound guidance.

Considering the similarities of commercially available toxins, it is reasonable that both abobotulinum and onabotulinum toxin $\mathrm{A}$ would provide analogous results [11].

Injections were performed under ultrasound guidance to localize precisely BTX spreading into salivary glands and to prevent diffusion on contiguous muscles that may produce further swallowing difficulties. Just in one case dysphagia became more severe for a few weeks after the treatment with no other complications. The remaining four patients did not report any adverse event following BTX injections.

In order to assess a possible effect of BTX treatment for sialorrhea on other clinical features, oral motor and upper respiratory functions were investigated using three selected items of RARS $[8,9]$. Eating habits and bruxism significantly improved in the weeks following treatment with BTX and worsened again when the toxin effect was wearing off. Therefore, saliva reduction may also give benefits on different clinical aspects. Eating could become indeed easier for patients having less retention of saliva. Interestingly, bruxism also improved after drooling reduction. We could speculate that an excessive amount of saliva may enhance jawclosing movements; therefore, a decrease in salivation may improve bruxism. However, a masticatory muscle relaxation due to BTX spreading from parotid glands can not be excluded [12]. Unfortunately, our study did not show a correlation between drooling production and respiratory performances, although we 
found a slight tendency of dyspnoea improvement after BTX treatment.

Results of the present study are restricted just to the first treatment with incobotulinumtoxin A. Actually, BTX injections can be performed recurrently with efficacy and safely [13] in different neurological disorders and it is reasonable that patients with RS can be treated regularly in case of excessive drooling.

Hypersalivation in RS is likely related to severe swallowing difficulties and contributes to complication of oral motor functions. This study is limited by the small number of patients, but our results would suggest that BTX injection in salivary glands is safe and effective in reducing saliva production in RS. Moreover, patients would also benefit from the treatment for more oral motor problems possibly related to hypersalivation like eating difficulties and bruxism. More extensive studies are needed to confirm these data. In conclusion, BTX treatment for sialorrhea in patients with RS should be encouraged because it is effective and safe and it may also improve important oral motor functions.

\section{ACKNOWLEDGEMENTS}

We thank the patients' families for giving us permission to report their daughter's medical history.

Funding. No funding or sponsorship was received for this study or publication of this article. The article processing charges were funded by the authors.

Authorship. All named authors meet the International Committee of Medical Journal Editors (ICMJE) criteria for authorship for this article, take responsibility for the integrity of the work as a whole, and have given their approval for this version to be published.

Disclosures. Pia Bernardo, Enza Raiano, Gerarda Cappuccio, Raffaele Dubbioso, Carmela Bravaccio, Emilia Vergara, Silvio Peluso, Fiore
Manganelli and Marcello Esposito have nothing to disclose.

Compliance with Ethics Guidelines. All procedures performed were in accordance with standards of the local Ethical Committee (Federico II University of Naples) and with the Helsinki Declaration of 1964, as revised in 2013. Informed consent was obtained from the legal representative of each patient included in the study.

Open Access. This article is distributed under the terms of the Creative Commons Attribution-NonCommercial 4.0 International License (http://creativecommons.org/licenses/ by-nc/4.0/), which permits any noncommercial use, distribution, and reproduction in any medium, provided you give appropriate credit to the original author(s) and the source, provide a link to the Creative Commons license, and indicate if changes were made.

\section{REFERENCES}

1. Neul JL, Kaufmann WE, Glaze DG, et al. Rett syndrome: revised diagnostic criteria and nomenclature. Ann Neurol. 2010;68:944-50.

2. Fehr S, Bebbington A, Nassar N, et al. Trends in the diagnosis of Rett syndrome in Australia. Pediatr Res. 2011;70:313-9.

3. Glaze DG, Percy AK, Skinner S, et al. Epilepsy and the natural history of Rett syndrome. Neurology. 2010;74:909-12.

4. Iodice R, Dubbioso R, Topa A, et al. Electrophysiological characterization of adult-onset NiemannPick type C disease. J Neurol Sci. 2015;348:262-5.

5. Coleman M, Brubaker J, Hunter K, Smith G. Rett syndrome: a survey of North American patients. J Ment Defic Res. 1988;32:117-24.

6. Reddihough D, Erasmus CE, Johnson $\mathrm{H}$, et al. Botulinum toxin assessment, intervention and aftercare for paediatric and adult drooling: international consensus statement. Eur J Neurol. 2010;17:109-21.

7. Thomas-Stonell N, Greenberg J. Three treatment approaches and clinical factors in the reduction of drooling. Dysphagia. 1988;3:73-8. 
8. Fabio RA, Martinazzoli C, Antonietti A. Costruzione e standardizzazione dello strumento "R.A.R.S." (Rett Assessment Rating Scale). Ciclo Evol Disabil. 2005;8:257-81.

9. Fabio RA, Billeci L, Crifaci G, et al. Cognitive training modifies frequency EEG bands and neuropsychological measures in Rett syndrome. Res Dev Disabil. 2016;53-54:73-85.

10. Blasco PA, Allaire JH. Drooling in developmentally disabled: management practices and recommendations. Consortium on drooling. Dev Med Child Neurol. 1992;34:849-62.
11. Kutschenko A, Manig A, Reinert MC, Mönnich A, Liebetanz D. In-vivo comparison of the neurotoxic potencies of incobotulinumtoxinA, onabotulinumtoxinA, and abobotulinumtoxinA. Neurosci Lett. 2016;3(627):216-21.

12. Tinastepe N, Küçük BB. Oral K. Botulinum toxin for the treatment of bruxism. Cranio. 2015;33:291-8.

13. Esposito M, Dubbioso R, Apisa P, Allocca R, Santoro L, Cesari U. Spasmodic dysphonia follow-up with videolaryngoscopy and voice spectrography during treatment with botulinum toxin. Neurol Sci. 2015;36:1679-82. 